\title{
Acute Pancreatitis and COVID-19: A Single-Center Experience
}

\author{
Anna Annunziata (D) \\ Antonietta Coppola' \\ Paolo Andreozzi ${ }^{2}$ \\ Maurizia Lanza' \\ Francesca Simioli ${ }^{\prime}$ \\ Novella Carannante ${ }^{3}$ \\ Camilla Di Somma' \\ Pierpaolo Di Micco ${ }^{4}$ \\ Giuseppe Fiorentino (D) \\ 'Department of Critic Area, I Unit \\ COVID, Monaldi-Cotugno Hospital, \\ Naples, Italy; ${ }^{2}$ Endoscopy and \\ Gastroenterology Unit, Marcianise \\ Hospital, Asl Caserta, Caserta, Italy; \\ ${ }^{3}$ First Division Infectious Disease, \\ Cotugno Hospital, Naples, Italy; ${ }^{4} \mathrm{UOC}$ \\ Internal Medicine, Fatebenefratelli \\ Hospital, Naples, Italy
}

\begin{abstract}
Gastrointestinal involvement in SARS-CoV-2 disease (COVID-19) can occur and evolve fatally. Reports are emerging that SARS-CoV-2 virus attacks the pancreatic cells, causing the boost of amylase and lipase serum activity and rarely frank pancreatitis. We retrospectively assessed all the patients admitted to the respiratory sub-intensive care and evaluated pancreatitis cases and their course. In our study, we included all patients admitted to our respiratory sub-intensive care unit from 1st to 30th November. All patients had a confirmed diagnosis of COVID-19 and a CT finding of interstitial pneumonia associated with signs of respiratory failure. We observed the course and evaluated who developed acute pancreatitis according to standard definitions. In this study, etiology of acute pancreatitis was defined on the basis of risk factors (ie, biliary pancreatitis was defined in presence of common bile duct stone or sludge at CT or MR). According to the Revised Atlanta Classification, we diagnosed and classified the patients and evaluated the radiological severity according to the Balthazar index and a computed tomography severity index. We found that $19 \%$ (15 of 78 patients) met the criteria for acute pancreatitis. The mortality rate among patients with pancreatitis was $20 \%$. Interestingly, in our population, cholelithiasis' imaging findings were found in only $7 \%$ of the patients, whereas no patient-reported alcohol consumption. Considering that alcohol and biliary stones represent the two major causes of AP in the general population, it is reasonable to hypothesize that SARS-CoV-2 could play a role in the etiology of acute pancreatitis in a subgroup of these patients.
\end{abstract}

Keywords: COVID-19, gastrointestinal involvement, ARDS

\section{Introduction}

Digestive symptoms can occur in patients with COVID-19 without respiratory signs. ${ }^{1}$ The SARS-CoV-2 disease involves the gastrointestinal tract through different mechanisms. Firstly, the virus gains cellular entry by ACE2 receptors expressed in both the gastrointestinal and respiratory tract epithelium, creating the potential for viral replication in the gastrointestinal system. ${ }^{2}$ Secondly, the gastrointestinal system could be damaged by the inflammatory response. SARS-CoV-2 could infect and destroy absorptive enterocytes, causing alteration of the intestinal secretion, malabsorption, and an activated enteric nervous system follow on symptoms like diarrhea. ${ }^{3}$ These patients had specific laboratory abnormalities related to hepatic injury - the pathophysiology behind SARS-CoV-2 infection may suggest that the injury is due to the disease process. $^{1-3}$ The real mechanism of liver involvement in SARS-CoV-2 infection is unclear. ${ }^{4}$ SARS-CoV-2 uses ACE 2 receptor to get into cells, as it has been established. This receptor is highly expressed in gastrointestinal epithelial cells, and the virus could infect cholangiocytes using this receptor and damages liver function. ${ }^{1,4}$
Correspondence: Antonietta Coppola Email antonietta.coppola84@gmail.com 
Moreover, ACE2 expression in liver tissue is augmented during the compensatory proliferation of hepatocytes derived from bile duct epithelial cells, contributing to hepatic damage. ${ }^{2}$ Furthermore, immune-mediated inflammation, hypoxia due to pneumonia and cytokine storm contribute to hepatocellular injury or liver failure in critically ill COVID19 patients. ${ }^{5}$ Lastly, some medications used in COVID-19 patients such as remdesivir (an RNA polymerase inhibitor) and hydroxychloroquine could determine drug-induced hepatotoxicity with the increase of liver enzymes. In literature, some recent publications have reported as SARS-CoV -2 could target pancreatic cells, causing elevation of serum lipase activity and rarely frank pancreatitis. ${ }^{9-12}$ However, it is conceivable that SARS-CoV-2 targets adipocytes, leading to increased lipolysis through adipose triglyceride lipase, ${ }^{13,14}$ contributing to pancreatic damage.

\section{Materials and Methods}

We retrospectively examined 78 patients who accessed the sub-intensive respiratory care unit of Cotugno hospital in Naples during November 2020 and their characteristics are reported in Table 1. Among them, we evaluated how many met the acute pancreatitis diagnostic criteria according to the Revised Atlanta Classification, and we also assessed the severity score considering the presence of single- or multiple-organ failures (persistent $>48 \mathrm{~h}$ ); we did not consider respiratory failure. We evaluated the following parameters:

Table I Patients' Characteristics

\begin{tabular}{|c|c|}
\hline & $\begin{array}{c}\text { Acute } \\
\text { Pancreatitis } \\
(n=15)\end{array}$ \\
\hline Age, mean $\pm S D$ & $60.5 \pm 11.8$ \\
\hline Male sex, n (\%) & $14(93.3)$ \\
\hline BMI, mean $\pm S D$ & $29.6 \pm 4.3$ \\
\hline Alcohol consumption, n (\%) & I (6.7) \\
\hline Cholelithiasis, n (\%) & $\mathrm{I}(6.7)$ \\
\hline Previous acute pancreatitis, n (\%) & $0(0)$ \\
\hline Ranson score, mean $\pm S D$ & $2.8 \pm 1.6$ \\
\hline Ranson score $\geq 3$, n (\%) & $9(60.0)$ \\
\hline Organ failure, $n(\%)$ & $4(26.7)$ \\
\hline Mortality, n (\%)* & $3(20.0)$ \\
\hline \multirow{2}{*}{\multicolumn{2}{|c|}{$\begin{array}{l}\text { Diagnostic criteria according to the Revised } \\
\text { Atlanta Classification }\end{array}$}} \\
\hline & \\
\hline $\begin{array}{l}\text { Abdominal pain, } \mathrm{n}(\%) \\
\text { Serum lipase or amylase level greater than }\end{array}$ & $9(60.0)$ \\
\hline $\begin{array}{l}\text { Serum lipase or amylase level greater than } \\
\text { three times the upper normal value, } n(\%)\end{array}$ & II (73.3) \\
\hline Characteristic imaging findings, n (\%) & $15(100)$ \\
\hline
\end{tabular}

Note: *Respiratory failure not considered. presence of abdominal pain, serum lipase or amylase level greater than three times the upper normal value, and characteristic imaging findings. All patients underwent an abdominal ultrasound and abdominal CT with contrast medium. We calculated the Balthazar index and a computed tomography severity index, and we evaluated the course of pancreatitis and patient outcome. We evaluated the mortality rate among patients with acute pancreatitis. The Ranson score was evaluated as a prognostic indicator: at time zero, the following points were evaluated: age $>55$ years, white blood cells count $>16,000 \mathrm{dL}, \mathrm{LDH}>350 \mathrm{U} /$ $\mathrm{L}$, aspartate aminotransferase $(\mathrm{AST})>250 \mathrm{U} / \mathrm{L}$, glycemia $>250 \mathrm{mg} / \mathrm{dL}$; after $48 \mathrm{~h}$, we recorded the presence of urea higher than $5 \mathrm{mg} / \mathrm{dL}$, a base deficit $>4 \mathrm{mEq} / \mathrm{L}$ on blood gas analysis, hematocrit reduced by $10 \%$ or more, hypoxia with $\mathrm{pO}_{2}<60 \mathrm{mmHg}$, serum calcium $<8 \mathrm{mg} / \mathrm{dL}$, and sequestration of liquids with dehydration $>6$ liters.

\section{Results}

Fifteen patients (19\%) were diagnosed with acute pancreatitis. The mean age of patients diagnosed with pancreatitis was $60.5 \pm 11.8$ years, including 14 (93.3\%) males and 1 female (Table 1). BMI (mean \pm SD) was 29.6 \pm 4.3 . One patient presented with a history of previous cholelithiasis, but no patients had previous acute pancreatitis, and no patients consumed alcohol. The Ranson score was $2.8 \pm 1.6$ (mean $\pm \mathrm{SD})$; nine patients $(60.0 \%)$ presented a Ranson score $\geq 3$ (probability of mortality greater than $16 \%)$. We recorded abdominal pain in nine patients $(60 \%)$. Eleven patients $(73.3 \%)$ had serum amylase or lipase level greater than three times the upper normal value, and we found characteristic imaging findings in 15 subjects $(100 \%)$. The severity of acute pancreatitis was stratified according to the Revised Atlanta Classification. Three patients had mild acute pancreatitis, and 12 patients $(80 \%)$ had severe acute pancreatitis. Organ failure was observed in 4 out of 15 patients (26.7\%). Only one patient had evidence of cholelithiasis. The radiological severity of pancreatitis was calculated according to the Balthazar index. Six patients (40\%) were A, six patients $(40 \%)$ were B-C, and three patients $(20 \%)$ were D-E. The computed tomography severity index was mild (0-3) for 12 patients $(80 \%)$, moderate (4-6) for 2 patients, and severe $(7-10)$ for one patient (Table 2). Three patients died. Twenty patients, all males, had pancreatic enzyme abnormalities without a diagnosis of pancreatitis. 
Table 2 Severity of Acute Pancreatitis

\begin{tabular}{|l|c|}
\hline & $\begin{array}{c}\text { Acute Pancreatitis } \\
(\mathbf{n}=\mathbf{1 5})\end{array}$ \\
\hline Balthazar index & $6(40)$ \\
A, $\mathrm{n}(\%)$ & $6(40)$ \\
B-C, $\mathrm{n}(\%)$ & $3(20)$ \\
D-E, $\mathrm{n}(\%)$ & \\
\hline Computed tomography severity index & $12(80)$ \\
Mild (0-3) & $2(13.3)$ \\
Moderate (4-6) & $1(6.7)$ \\
Severe (7-10) & \\
\hline Severity of acute pancreatitis according to & \\
the Revised Atlanta Classification* & $3(20)$ \\
Mild acute pancreatitis, $\mathrm{n}(\%)$ & $0(0)$ \\
Moderately severe acute pancreatitis, $\mathrm{n}(\%)$ & $12(80)$ \\
\hline Severe acute pancreatitis, $\mathrm{n}(\%)$ & \\
\hline
\end{tabular}

Note: *Respiratory failure not considered.

\section{Discussion}

In our group of 78 patients, we observed that $15(19 \%)$ had acute pancreatitis, and among them, $12(80 \%)$ patients had severe acute pancreatitis. Twenty patients had pancreatic enzyme elevation without a diagnosis of pancreatitis. No patients had diabetes mellitus, and they did not use drugs with pancreatic toxicity, except for non-steroidal antiinflammatory drugs (NSAIDs) that they used before admission to the hospital. Only one patient presented a cholelithiasis history, and no patients had previous acute pancreatitis or used alcohol. In this group, therefore, no patients had risk factors for the development of pancreatitis. Interestingly, no patients reported gastroenteric symptoms, except for epigastric pain, or a had history of gastrointestinal symptoms suggestive of chronic pancreatitis.

Pancreatic involvement in viral infections is common. The most-reported viruses with pancreatic tropism are hepatitis A, B and E virus, Epstein-Barr virus, coxsackievirus, cytomegalovirus, herpes zoster virus, HIV, and viruses causing mumps, measles, and varicella-zoster. Every virus has a different mechanism of viral pancreatic injury. ${ }^{6}$ SARS-CoV-2 affects the pancreas to varying degrees. Wang et $\mathrm{al}^{7}$ examined 52 patients with COVID19 pneumonia. They showed that $17 \%$ of the patients experienced pancreatic damage that consists in defined abnormality in amylase or lipase levels ${ }^{7}$ without clinical symptoms of severe pancreatitis. The mechanism is not clear. The ACE2 receptor is expressed in pancreatic islet cells, so SARS-CoV-2 infection can theoretically damage pancreatic islet leading in acute diabetes. Mechanisms of pancreatic injury could include indirect damage and indirect damage. SARS CoV-2 can cause a direct cytopathic effect on pancreatic cells, or cause indirect damage by systemic inflammatory and immune-mediated response, leading to organ damage and elevated pancreatic enzyme levels. ${ }^{7,8}$ Antipyretics are often used by patients in this study prior to admission, causing a drug-related pancreatic injury. ${ }^{8}$ Moreover, all patients had severe hypoxaemia due to bilateral pneumonia that required non-invasive ventilation, which can lead to hypoxic pancreatic injury. A recent meta-analysis compared 12 studies $^{15}$ and showed that in severe acute pancreatitis and COVID-19, the pattern of altered cytokine levels is very similar. Thus, IL-6, IL-8, and IL-10 serum levels were higher in severe cases with respectcompared with those in non-severe cases of the two diseases. A comparable behaviour was observed for tumor necrosis factor- $\alpha$, whereas no differences were observed for interferon- $\gamma$ levels as a marker of severity. Severity is determined by both the extent of pancreatic inflammation and systemic inflammation. Scores such as the Balthazar index and the computed tomography severity index assess pancreatic inflammation, while the Atlanta classification considers both pancreatic inflammation and systemic involvement.

In our study, we had radiologically mild pancreatitis but a high rate of systemic complications. The pathogenic mechanisms triggered by SARS-CoV-2 infection probably involve the pancreas, in predisposed individuals, and other organs. In addition, we have to consider the presence of respiratory failure present in all patients that can cause hypoxemic pancreatic damage. On the other hand, in our population, cholelithiasis' imaging findings were found in only $7 \%$ of the patients, whereas no patients reported alcohol consumption. Considering that alcohol and biliary stones represent the two major causes of acute pancreatitis in the general population, it is conceivable to hypothesise that SARS-CoV-2 could have a role in the aetiology of acute pancreatitis in a subgroup of patients. Mortality among patients with pancreatitis etiology\% (3 out of 15 patients), but pancreatitis was the cause of death for only one patient. In our sub-intensive unit, the overall mortality in November 2020 was $11.5 \%$ (9 of 78 patients). We observed higher mortality in patients with acute pancreatitis $(20 \%)$ compared to patients without acute pancreatitis (9.5\%). However, this difference was not significant, and results suggest that acute pancreatitis may worsen patients' 
prognosis with COVID-19 pneumonia. In a retrospective study Gubatan and other authors found a higher prevalence of COVID-19 in patients with a history of acute and chronic pancreatitis, suggesting that patients with a history of pancreatitis may be more susceptible to COVID-19. ${ }^{16}$ We do not have collected data regarding previous episodes of acute pancreatitis. However, if COVID-19 plays a role in the pancreatic inflammation, it is reasonable to hypothesize that patients with risk factors (ie, stones, alcohol) may experience acute pancreatitis during the infection.

In conclusion, gastrointestinal involvement in SARSCoV-2 infection is very common and conservatively managed. In this regard, the use of all drugs that can further damage pancreatic function, such as high-dose steroids, should be carefully evaluated. Antibiotics administration is controversial and is recommended only if there are signs of bacterial infection. According to our experience, it is important to monitor pancreatic parameters even on a routine basis, before the onset of acute symptoms, to prevent dramatic complications. However, our considerations are deduced from observing a limited number of cases in a single center. Further evidence is needed to ascertain whether pancreatic damage can significantly affect patients' clinical course and mortality COVID-19.

\section{Ethics and Consent Statements}

Institutional review board statement: approved by the ethics committee of University of Campania "Luigi Vanvitelli"- AORN "Ospedali dei colli" with number $2020 / 485$ on November 26, 2020. The guidelines outlined in the Declaration of Helsinki were followed. All subjects provided informed consent.

\section{Disclosure}

The authors report no conflicts of interest in this work.

\section{References}

1. Patel KP, Patel PA, Vunnam RR, et al. Gastrointestinal, hepatobiliary, and pancreatic manifestations of COVID-19. J Clin Virol. 2020;128:104386. PMID: 32388469; PMCID: PMC7189193. doi:10.1016/j.jcv.2020.104386
2. Pan L, Mu M, Yang P, et al. Clinical characteristics of COVID-19 patients with digestive symptoms in Hubei, China: a Descriptive, Cross-Sectional, Multicenter Study. Am J Gastroenterol. 2020;115 (5):766-773. PMID: 32287140; PMCID: PMC7172492. doi:10.14309/ajg.0000000000000620

3. Zhang H. The digestive system is a potential route of 2019-nCov infection: a bioinformatics analysis based on single-cell. Biorxiv. 2020. doi:10.1101/2020.01.30.927806

4. Wong SH, Lui RN, Sung JJ. Covid-19 and the digestive system. J Gastroenterol Hepatol. 2020;35(5):744-748. doi:10.1111/jgh.15047

5. Zhang C, Shi L, Wang F-S. Liver injury in COVID-19: management and challenges. Lancet Gastroenterol Hepatol. 2020;5(5):428-430. doi:10.1016/s2468-1253(20)30057-1

6. Rawla P, Bandaru S, Vellipuram A. Review of infectious etiology of acute pancreatitis. Gastroenterol Clin North Am. 2017;10(3):153.

7. Wang F, Wang H, Fan J, Zhang Y, Wang H, Zhao Q. Pancreatic injury patterns in patients with COVID-19 pneumonia. Gastroenterology. 2020. doi:10.1053/j.gastro.2020.03.055

8. Liu F, Long X, Zhang B, Zhang W, Chen X, Zhang Z. ACE2 expression in pancreas may cause pancreatic damage after SARS-CoV-2 Infection. Clin Gastroenterol Hepatol. 2020;18 (9):2128-2130.e2. PMID: 32334082; PMCID: PMC7194639. doi:10.1016/j.cgh.2020.04.040

9. McNabb-Baltar J, Jin DX, Grover AS, et al. Lipase elevation in patients with COVID-19. Am J Gastroenterol. 2020;115 (8):1286-1288. PMID: 32496339; PMCID: PMC7288768. doi:10.14309/ajg.000000000000732

10. Bruno G, Fabrizio C, Santoro CR, Buccoliero GB. Pancreatic injury in the course of coronavirus disease 2019: a not-so-rare occurrence. J Med Virol. 2020:10.1002/jmv.26134. PMID: 32497298; PMCID: PMC7300736. doi:10.1002/jmv.26134

11. Thaweerat W. Current evidence on pancreatic involvement in SARS-CoV-2 infection. Pancreatology. 2020;20(5):1013-1014. PMID: 32498973; PMCID: PMC7251394. doi:10.1016/j. pan.2020.05.015

12. Hadi A, Werge M, Kristiansen KT, et al. Coronavirus disease-19 (COVID-19) associated with severe acute pancreatitis: case report on three family members. Pancreatology. 2020;20(4):665-667. PMID: 32387082; PMCID: PMC7199002. doi:10.1016/j. pan.2020.04.021

13. El-Kurdi B, Khatua B, Rood C, et al. Mortality from coronavirus disease 2019 increases with unsaturated fat and may be reduced by early calcium and albumin supplementation. Gastroenterology. 2020;159(3):1015-1018.e4. doi:10.1053/j.gastro.2020.05.057

14. de Oliveira C, Khatua B, Noel P, et al. Pancreatic triglyceride lipase mediates lipotoxic systemic inflammation. J Clin Invest. 2020;130 (4):1931-1947. PMID: 31917686; PMCID: PMC7108918. doi:10.1172/JCI132767

15. Hegyi P, Szakács Z, Sahin-Tóth M. Lipotoxicity and cytokine storm in severe acute pancreatitis and COVID-19. Gastroenterology. 2020;159(3):824-827. PMID: 32682765; PMCID: PMC7366088. doi:10.1053/j.gastro.2020.07.014

16. Gubatan J. Prevalence, risk factors and clinical outcomes of COVID-19 in patients with a history of pancreatitis in northern California. Gut. 2021;70(2):440-441. 


\section{Publish your work in this journal}

The Journal of Multidisciplinary Healthcare is an international, peerreviewed open-access journal that aims to represent and publish research in healthcare areas delivered by practitioners of different disciplines. This includes studies and reviews conducted by multidisciplinary teams as well as research which evaluates the results or conduct of such teams or healthcare processes in general. The journal covers a very wide range of areas and welcomes submissions from practitioners at all levels, from all over the world. The manuscript management system is completely online and includes a very quick and fair peer-review system. Visit http://www.dovepress.com/testimonials. php to read real quotes from published authors. 